\title{
Efficient Broadcasting of Safety Messages in Multihop Vehicular Networks
}

\author{
Carla-Fabiana Chiasserini ${ }^{1}$, Rossano Gaeta ${ }^{2}$, Michele Garetto $^{1}$, Marco Gribaudo $^{2}$, and Matteo Sereno ${ }^{2}$ \\ ${ }^{1}$ Politecnico di Torino \\ Dipartimento of Elettronica \\ 10129 Torino, Italy \\ \{chiasserini,garetto\}@polito.it \\ ${ }^{2}$ Università di Torino \\ Dipartimento di Informatica \\ 10129 Torino, Italy \\ \{rossano,marcog,matteo\}@di.unito.it
}

\begin{abstract}
We focus on a vehicular network supporting safety applications, and we present an application and a channel access mechanism for efficient multihop broadcasting. We study the performance of the proposed solution by developing an analytical framework, which provides several metrics relevant to message dissemination. Analytical results are compared with the performance obtained through ns.
\end{abstract}

\section{Introduction}

Transportation safety is one of the most important applications of vehicular networks. Vehicles can communicate information on traffic and road conditions with each other, as well as with fixed network nodes. Examples are warning messages that are generated by approaching emergency vehicles, cars that stopped or vehicles stuck in a road tunnel because of an accident. Typically, safety messages need to be broadcasted to all vehicles traveling over a geographical area, and need to be delivered with high reliability and low delay. It is therefore important to develop protocol solutions that meet such requirements.

Broadcast solutions at the MAC layer are studied in [2, 1, 3]. In [1], an IEEE 802.11-based scheme is proposed to address the broadcast storm and the hidden terminal problems in urban areas. The use of IEEE 802.11e EDCA scheme for priority access is investigated in [2], where the authors study through simulation the broadcast reception rate in presence of different channel propagation models. The performance of the optimum broadcast algorithm defined over the node minimum connected dominating set is studied in [3], in the case of a unidimensional ad hoc network.

This work was supported by the Italian Ministry for Scientific Research through the PATTERN project.
In this work we study the dissemination of safety messages over vehicular networks, on road segments where there are not fixed nodes and dissemination relies on ad hoc communications only. We present a channel access scheme and an application mechanism that provide an efficient multihop broadcasting. We introduce a spatial differentiation approach at the MAC layer: vehicles that are about to rebroadcast the message access the channel with different priority, depending on their distance from the last vehicle that transmitted. This technique reduces the broadcast delay along the road, thus improving the message timeliness. Also, we develop an analytical framework to study the system performance, and derive several metrics relevant to the dissemination of safety messages.

\section{System Description}

We focus on a unidimensional inter-vehicular network, modeling a highway or a tunnel, i.e., a traffic scenario where safety applications are of paramount importance. Vehicles travel over a single-lane road, and they are randomly distributed with spatial density that may vary along the road. Note that our model can be easily extended to deal with the case of a multi-lane road (omitted here for the lack of room); also the extension to the two-dimensional case is straightforward when the system behavior on different road segments can be assumed to be independent of each other.

We look at a snapshot of the traffic stream; indeed, considering typical values for the vehicle speeds, the message length and the communication data rates, it results that the vehicle movement during a message broadcasting is negligible. We therefore assume that vehicle positions remain constant during the message forwarding over the whole road.

As an example, consider the 802.11 technology. By fixing the message length at 32 bytes, the data rate at $11 \mathrm{Mb} / \mathrm{s}$ and the contention window at 31 , the average time to forward a broadcast message over one hop is about $522 \mu \mathrm{s}$. Assuming a vehicle speed of $100 \mathrm{~km} / \mathrm{h}$, during the message forwarding time vehicles move $0.014 \mathrm{~m}$. 
All vehicles have a common coverage radius, equal to $R$. Also, vehicles are equipped with a GPS device and include their position in each transmitted message.

Finally, we focus on safety applications and neglect other kinds of data traffic. This is justified by the fact that, if other applications are simultaneously supported by the network, safety messages will have higher priority and their transmission on the wireless medium should not be affected by other types of traffic.

\section{Dissemination of Safety Messages}

Here we propose a channel access scheme and an application that aim at reducing the delay and the overhead of the safety broadcast. Our channel access scheme provides channel access priority by exploiting the concept of spatial differentiation, while the safety application is based on the vehicle ability to detect the message direction of arrival.

The Spatial Differentiation Approach. At the MAC layer, we envision an access scheme based on the CSMA/CA mechanism (e.g., based on the IEEE 802.11 standard). The binary exponential backoff procedure is employed, and the backoff time is a number $b$ of slot time intervals of duration $\sigma$. We have that $b$ is a random number uniformly distributed over $[0, W]$, where $W$ is the contention window. Also, we consider that, whenever the MAC layer receives a message from the higher layers, it extracts a backoff value, so that a random time interval is waited before attempting to access the channel.

Our key idea is to assign different access priorities to the vehicles that are currently in charge of forwarding the message, so that the advancement corresponding to a message hop is maximized.

Let $v$ be the last vehicle that (re)broadcasted the message. We define different forwarding zones within the coverage range of $v$, and assign to the vehicles belonging to each zone a different value of contention window. The larger the distance from the sender $v$, the smaller the contention window. By doing so, vehicles belonging to the the furthest zone have the highest priority in accessing the channel, and the probability that the message forwarding is performed by vehicles at distance close to the coverage radius of the previous sender is increased.

Note that such differentiation mechanism could be implemented through the IEEE 802.11e technology. Indeed, the traffic transmitted by vehicles belonging to different zones could be mapped onto different 802.11e Access Categories, based on the geographical position of the vehicle.

The Safety Application. Consider a vehicle wishing to broadcast a safety message along the road; we define this vehicle as the broadcast message source. The application we devise allows for a smart broadcasting technique that, based on the vehicle ability to determine the direction of arrival of the message, reduces the communication overhead.

The procedure followed by each vehicle application along the road (excluding the source) is presented below.

(i) Upon the reception of the safety message, the application first checks whether the message is received for the first time and its direction of arrival.

(ii) If the message has never been received before, the application passes the message to the MAC layer to rebroadcast the information. Recall that a random backoff time is waited at the MAC layer before accessing the channel.

(iii) If it is a duplicated message, the application flushes out the previously received copy (which is buffered at the MAC layer waiting to be transmitted) and processes the newly arrived copy. The new copy will be either passed to the MAC layer or discarded, based on its direction of arrival. It will be passed to the MAC layer if its direction of arrival is the same as the one of the original message; it will be discarded otherwise.

Note that, according to this procedure, a vehicle that detects the message being rebroadcasted further ahead, will abandon its transmission attempt, avoiding unnecessary message forwarding. Furthermore, as the message propagates along the road, a vehicle may receive multiple copies of the message from different vehicles located on its left side. In this case the distance between the vehicle and the last message sender decreases progressively. This implies that, at every message reception, a vehicle needs to start a new transmission attempt with an updated value of contention window. Our broadcast scheme meets this requirement and dynamically adapts to the message advancements, since the application always processes the newly arrived copy and discards the previous one.

\section{Assumptions and Notations}

Let us consider a single-lane road; we use the minimum distance $\Delta$ between two vehicles to discretize space along the lane. It follows that vehicles can occupy only a discrete set of positions indexed by $y$, with $y \in \mathbb{N}$, and a vehicle at position $y$ is at distance $y \Delta$ from the origin. As mentioned before, the extension to the multi-lane case can be easily done.

The normalized coverage radius $r=R / \Delta$ is the maximum number of vehicles receiving the message on either side of a transmitter. For the sake of simplicity, we assume that the source of the broadcast message is located at $y=0$ and consider the message broadcasting only on one side of the source. The extension to the case where the source is 
located at $y>0$, and the message broadcasting occurs on both sides of the source, is straightforward. We define the occupation probability $\rho_{i}$ as the probability that position $i$ is occupied by a vehicle (with $i>0$ ); note that the vehicle density can be expressed as $\rho_{i} / \Delta$.

We discretize time into slots of duration $\sigma$. The duration of a broadcast message is set to $T$ slots, and the message originates from the source at time $n=0$ (i.e., the first bit of the message is placed on the channel at time zero). The backoff time of a vehicle is uniformly distributed in $\left[0, W_{j}\right]$, with $j$ being the vehicle distance from the last message sender and $W_{j}$ the contention window expressed in time slots.

At the physical layer, we make the following assumptions: (i) there is perfect capture, meaning that a vehicle simultaneously receiving more than one message at a time is able to lock on the strongest signal and receive the message correctly; (ii) the radio channel is error-free. We verified through simulation that the impact of the perfect capture assumption is acceptable in our system scenario.

\section{Metrics of interest}

Since broadcasting of safety messages poses strict requirements in terms of reliability and message timeliness, we consider the following metrics.

Message block probability $P_{B}[y]$ : the probability that the message broadcast stops at the vehicle occupying position $y$, i.e., the probability that the message will not be delivered to any other vehicle beyond $y$.

First reception probability $P_{R}[y, n, h]$ : the probability that a vehicle at position $y$ receives for the first time the message at time $n$, in $h$ hops. From $P_{R}[y, n, h]$ it is possible to derive the marginal probability that a vehicle in $y$ receives the message for the first time at $n$, regardless of the number of hops $\left(\bar{P}_{R}[y, n]\right)$, and the marginal probability of first reception in $y$, in $h$ hops $\left(\bar{P}_{R}[y, h]\right)$, regardless of the delay.

The message block probability is computed in Section 6 by using a static analysis, that is without the need to explicitly considering the temporal dynamics of the system.

The second metric, instead, requires a study of the temporal evolution of the message broadcast. An analysis of the transient behavior of the system, which provides $P_{R}[y, n, h]$ is presented in Section 7. To reduce the computation time of this important metric, in Section 7.1 we restrict ourself to the case of homogeneous occupation probability and introduce an approximated technique.

\section{Computation of the block probability}

Consider the case that the occupation probability is constant along the road (i.e., $\rho_{i}=\rho, \forall i>0$ ). We first de- rive the probability $P_{A}[y]$ that the furthest spatial position reached by the message transmission as time goes to infinity is $y$, i.e., the probability that the transmission carrying the broadcast message has covered a physical distance up to position $y$. By definition, we have: $P_{A}[0]=1$.

Probability $P_{A}[y]$ can be easily computed based on the following observations. Firstly, a message transmitted by a vehicle at position $x$ can be received up to position $x+r$. Thus, the message broadcast stops at $x$ only if there are no vehicles between $x+1$ and $x+r$. In other words, the message eventually arrives at the generic position $y$ if between 0 and $y$ there are no gaps in the vehicle distribution, of width greater than or equal to $r$. Secondly, computing the probability that there is connectivity between 0 and $y$ maps onto the known problem of finding the probability that, in a sequence of $y$ Bernoulli trials, there are no runs of length $r$. (This is due to the assumption that each position $i$ is independently occupied by a vehicle with given probability $\rho_{i}=\rho$.) It follows that the most efficient way to calculate $P_{A}[y]$, with $y>r+1$, is given by the following recursive equation:

$$
P_{A}[y]=P_{A}[y-1]-\rho(1-\rho)^{r} P_{A}[y-r-1]
$$

starting from the initial values: $P_{A}[i]=1,(0 \leq i \leq r)$ and $P_{A}[r+1]=1-(1-\rho)^{r}$. The above formula has the following intuitive explanation: a message transmission that has arrived up to position $y-1$ will reach also position $y$ unless $y-1$ is the last position that completes a series of $r$ empty positions (i.e., without vehicles).

It can be shown that in the case where the occupation probability varies along the road (i.e., inhomogeneous vehicle density), for any $y>r+1$ (1) becomes:

$$
\begin{aligned}
P_{A}[y]= & P_{A}[y-1]-\rho_{y-r-1} \prod_{y-r \leq j<y}\left(1-\rho_{j}\right) \\
& P_{A}[y-r-1]
\end{aligned}
$$

starting from the initial values: $P_{A}[0]=1 ; P_{A}[i]=\rho_{i},(1 \leq$ $i \leq r)$; and $P_{A}[r+1]=1-\prod_{1 \leq j \leq r}\left(1-\rho_{j}\right)$.

The block probability $P_{B}[y]$ can be computed as the probability that the message reaches the vehicle in $y$ and this is the last vehicle to receive the message. A vehicle in $y$ will be the last one to receive the message if its location is followed by a connectivity gap, which happens with probability $\prod_{j=y+1}^{y+r}\left(1-\rho_{j}\right)$. Thus, we have:

$$
P_{B}[y]=\rho_{y} P_{A}[y] \prod_{y+1 \leq j \leq y+r}\left(1-\rho_{j}\right)
$$

\section{Probability of first reception}

To evaluate the timeliness of the broadcast message delivery, we need to study the temporal dynamics of the mes- 
sage broadcasting along the road and to analyze the transient behavior of the system.

The dynamics of the message broadcasting are illustrated in Figure 1 (in the figure the arrows indicate the direction of the message broadcast). Suppose that at time $k$ vehicle $x$ starts sending the message. All vehicles in range of $x$ that were contending for the channel will suspend their transmission attempt for the duration $T$ of the message. At time $k+T$ the message is fully received by all vehicles up to position $x+r$. After that, the vehicles in $[x+1, x+r]$ contend among themselves to further forward the message, using a contention window that depends on their distance from $x$. The time spent in contention will be equal to the minimum backoff value among the contending vehicles. Notice that more than one vehicle can extract the minimum value of backoff, resulting in simultaneous transmissions at the end of the contention period; however the message will be successfully forwarded also in this case. Indeed, let $y$ be the position of the furthest vehicle transmitting the message after the contention period: thanks to the assumption of perfect capture and ideal wireless channel, after time $T$ the message sent by $y$ will be successfully received by all vehicles up to position $y+r$.

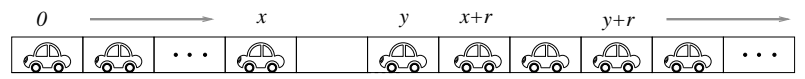

\section{Figure 1. Broadcasting dynamics}

In order to compute the probability of first reception $P_{R}[y, n, h]$, we introduce the following definitions. $P_{T}[y, n, h, l]$ is the transmission probability, i.e., the probability that a vehicle at position $y$ transmits the message at time $n$ in $h$ hops, having received the message from its predecessor at distance $l . \quad \bar{P}_{T}[y, n, h]$ is the marginal transmission probability regardless of the predecessor: $\bar{P}_{T}[y, n, h]=\sum_{l=1}^{r} P_{T}[y, n, h, l] . \mathbf{P}(S=y, C=$ $b, L=l)$ is the probability that a vehicle at position $y$ extracts a backoff value of $b$ slots, it wins the channel contention and the message advances by $l$ positions on the road (i.e., the length of the hop between $y$ and the location of the predecessor vehicle is equal to $l$ ).

We first write the transmission probabilities as:

$$
\begin{aligned}
P_{T}[y, n, h, l]= & \sum_{b=0}^{W_{l}}\left[\bar{P}_{T}[y-l, n-T-b, h-1]\right. \\
& \mathbf{P}(S=y, C=b, L=l)]
\end{aligned}
$$

for $y>0, n \geq T, h \geq 1$, and $1 \leq l \leq r$. The sum on the right hand side of (4) accounts for all possible values $b$ of backoff time that the vehicle in $y$ can extract. Also, note that the contention window used by the vehicle in $y$ will depend on $l$, being $l$ the distance between $y$ and its predecessor.
By definition, the marginal probability $\bar{P}_{T}[y, n, h]$ can be computed from (4) through the recursive equation:

$$
\begin{aligned}
\bar{P}_{T}[y, n, h]= & \sum_{l=1}^{r} \sum_{b=0}^{W_{l}}\left\{\bar{P}_{T}[y-l, n-T-b, h-1]\right. \\
& \mathbf{P}(S=y, C=b, L=l)\}
\end{aligned}
$$

The two sums on the right hand side of (5) account, respectively, for all possible pairs $(y-l, b)$ representing the position of the previous transmitter with respect to $y$ and the value $b$ of backoff time that the vehicle in $y$ can extract. The initial value of $\bar{P}_{T}[y, n, h]$ is: $\bar{P}_{T}[0,0,1]=1$, which accounts for the fact that the message source makes its transmission at time 0 , performing the first hop.

Next, we compute the joint probability $\mathbf{P}(S=y, C=$ $b, L=l$ ). For the sake of clarity, let us first present the case of occupation probability constant and equal to $1\left(\rho_{i}=\rho=1\right)$. We need to compute the probability that the contention period lasts for $b$ slots and the message advances by $l$ positions on the road. This event happens if and only if: $(i)$ the vehicle at distance $l$ from the vehicle transmitting the message in the previous hop extracts a backoff value equal to $b$; (ii) all vehicles at distance $j<l$ extract a backoff higher than or equal to $b$; (iii) all vehicles at distance $j>l$ (within transmission range) extract a backoff value strictly higher than $b$. In general, a vehicle at distance $j$ extracts a backoff value uniformly in the contention window $\left[0, W_{j}\right]$, where $W_{j}$ depends on the distance $j$ to allow for spatial differentiation. We have:

$$
\begin{aligned}
\mathbf{P}(S=y, C=b, L=l)= & \frac{1}{W_{l}+1} \prod_{j=1}^{l-1} \frac{W_{j}-b+1}{W_{j}+1} \\
& \prod_{j=l+1}^{r} \frac{W_{j}-b}{W_{j}+1}
\end{aligned}
$$

Note that the three factors on the right hand side of (6) account for the events $(i),(i i)$ and (iii) described above.

Similarly, we can compute $\mathbf{P}(S=y, C=b, L=l)$ when the occupation probability varies along the road (i.e., $0<\rho_{i} \leq 1$, with $i>0$ ). In this case we need to take into account that there may be no vehicle at distance $j$ : if so, that position does not contribute to the contention phase. Then, by using (5), we obtain $\bar{P}_{T}[y, n, h]$, from which we compute $P_{T}[y, n, h, l]$ using (4).

Finally, the probability of first reception $P_{R}(y, n, h)$ (with $y>r, k \geq 2 T, h \geq 1$ ) can be written as,

$$
\begin{aligned}
P_{R}(y, n, h)= & \rho_{y} \sum_{m=1}^{r}\left\{\bar{P}_{T}[y-m, n-T, h]+\right. \\
& \left.-\sum_{l=1}^{r-m} P_{T}(y-m, n-T, h, l)\right\}
\end{aligned}
$$

Equality holds due to the perfect capture capability of the vehicles. 
starting from the following initial values: $P_{R}(0,0,0)=1$, which accounts for the message source, and $P_{R}(i, T, 1)=$ $\rho_{i}$ for $0<i \leq r$. Referring to (7), we have that: $\rho_{y}$ accounts for the probability that a vehicle is present at position $y$, while the sum over $m$ considers all possible predecessors within distance $r$ from $y$, i.e., all vehicles from which the one in $y$ can receive the message. Given $m$, the probability that the message is received for the first time in $y$ due to the transmission of a vehicle at distance $m$, is equal to the probability that the vehicle at $y-m$ transmits the message, and the message has not been heard by $y$ before. That is, the vehicle in $y-m$ must have received the message from a predecessor whose distance from $y$ is greater than $r$. Figure 2 illustrates the case where a transmission performed by the vehicle at position $y-m-l$ reaches the vehicle in $y-m$ but not the one in $y$ (Figure 2.(a)). The case where a transmission performed at $y-m-l$ reaches both the vehicles (in $y-m$ and in $y$ ) is presented in Figure 2.(b).
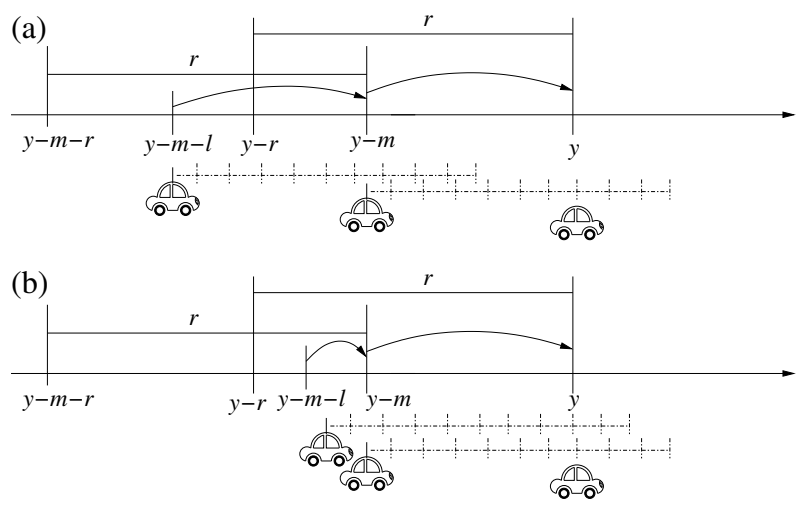

\section{Figure 2. Locations of the predecessor from which (a) a transmission is not received at $y$, (b) a transmission is received in $y$}

By summing over the number of hops ( $h \geq 1)$, we derive the probability $\bar{P}_{R}(y, n)$ that the vehicle at position $y$ receives the message for the first time at time $n$. By summing over time, we derive the probability $\bar{P}_{R}(y, h)$ that the message is received by a vehicle at position $y$ in $h$ hops. Note that it is also possible to derive the block probability similarly to (3): $P_{B}(y)=\sum_{n=0}^{\infty} \bar{P}_{R}(y, n) \prod_{y<i \leq y+r}\left(1-\rho_{i}\right)$.

\subsection{A Gaussian Approximation}

The analysis presented in the previous section is general enough to deal with an inhomogeneous vehicle density, however it requires to calculate the three-variable function $\bar{P}_{T}[y, n, h]$. Here we restrict ourself to the case of homogeneous vehicle density $\left(\rho_{i}=\rho, \forall i>0\right)$ and derive an approximate analysis of the system transient behavior that provides the marginal probability $\bar{P}_{R}[y, n]$ with low computational complexity. We first consider the case with $\rho_{i}=\rho=1, \forall i>0$, i.e., the situation in which all positions along the road are occupied by vehicles; the analysis in the case of occupation probability smaller than one is briefly sketched at the end of the section.

We start by observing that the message broadcasting along the road can be described by a sequence of independent hops having variable delay $D$ and variable length $L$ (with $L$ expressed in number of spatial intervals of length $\Delta$ ). The delay $D$ is the sum of a deterministic term (the message duration $T$ ) and the variable term $C$ given by the contention period. The maximum distance $M(n)$ reached by the message at time $n$ is therefore given by the sum of a random number $H(n)$ (the number of hops) of random variables $L$ (the hop length). By applying the central limit theorem, we can approximate the probability distribution of the furthest distance reached by the message at time $n$ with a normal distribution $P_{M}[y, n]$. To specify the normal distribution of $M(n)$, we need to compute mean and variance of $M(n)$. This can be done as follows.

We observe that from the joint probability $\mathbf{P}(S=y, C=$ $b, L=l)$ in (6), we can derive the marginal probability $P_{D}(d)$ that the total hop delay is equal to $d$, as well as the marginal probability $P_{L}(l)$ that the hop length is equal to $l$. Note that the dependence on $y$ drops due to the assumption of homogeneous occupation probability. We have:

$$
\begin{aligned}
& P_{D}(d)= \sum_{l=1}^{r} \mathbf{P}(S=y, C=d-T, L=l) \\
& T \leq d \leq T+W_{l} \\
& P_{L}(l)=\sum_{b=0}^{W_{l}} \mathbf{P}(S=y, C=b, L=l) \quad 1 \leq l \leq r
\end{aligned}
$$

From (8) we compute the mean hop delay $E[D]$ and its variance $\operatorname{Var}[D]$; from (9) we compute the first two moments of hop length $E[L]$ and $E^{2}[L]$. The average number of hops $E[H(n)]$ done at time $n$ is: $E[H(n)]=n / E[D]$. The variance $\operatorname{Var}[H(n)]$ of the number of hops can be expressed as: $\operatorname{Var}[H(n)]=\frac{\operatorname{Var}[D] n}{(E[D])^{3}}$. Then, it is possible to calculate $E[M(n)]$ and $\operatorname{Var}[M(n)]$ from the first two moments of $H(n)$ and $L$ as:

$$
\begin{aligned}
E[M(n)] & =E[H(n)] E[L] \\
\operatorname{Var}[M(n)] & =E[H(n)] \operatorname{Var}[L]+E^{2}[L] \operatorname{Var}[H(n)]
\end{aligned}
$$

The Gaussian approximation is more and more accurate as $n \rightarrow \infty$; in practice, it already produces satisfactory results after the message has propagated for a few hops (namely, 10 hops).

An approximated expression of $\bar{P}_{R}[y, n]$ can be obtained by considering that a vehicle in $y$ receives the message for the first time if its location falls within the spatial advancement of the last message hop, and such advancement occurs 
at time $n$. Since on average a message hop takes a time period of $E[D]$ and its average length is equal to $E[L]$, we write:

$$
\bar{P}_{R}[y, n] \approx P_{M}[y-E[L], n] \frac{E[L]}{E[D]}
$$

In the analysis above, we have neglected the fact that the first hop is special: it has a deterministic duration $T$ and advances by a deterministic length $r$. However this fact can be easily taken into account considering the contribution of the first hop separated from the rest.

The approximate analysis when $\rho \leq 1$ presents two additional complications: (i) the number of vehicles contenting for the channel at each hop is now randomly distributed; (ii) the message can be blocked at some point due to the lack of connectivity (see Section 6). We can first analyze the broadcast delay with $\rho \leq 1$ assuming that the network is connected from the source up to position $y$, and derive $\hat{P}_{M}[y, n]$. The probability $\hat{P}_{M}[y, n]$ can be easily obtained following the same approach as described above. Then, we decondition with respect to the assumption that there is connectivity from the source up to position $y$. To do so, we simply multiply probability $\hat{P}_{M}[y, n]$ by the probability $P_{A}[y]$ that the message indeed reaches position $y$. We therefore approximate $\bar{P}_{R}[y, n]$ as:

$$
\bar{P}_{R}[y, n] \approx \rho \hat{P}_{M}[y-E[L], n] P_{A}[y] \frac{E[L]}{E[D]}
$$

where $\rho$ is the probability that there is a vehicle in $y$.

\section{Performance Evaluation}

We evaluate the performance of the mechanisms proposed in Section 3, using the analytical framework, and we validate our models against detailed simulation experiments with $n s$.

We consider that the minimum distance between vehicles is $\Delta=5 \mathrm{~m}$. The normalized coverage radius is fixed to $r=9$. The MAC protocol considered in our experiments relies on the standard 802.11 DCF as implemented in $n s$. The backoff slot duration is $\sigma=20 \mu \mathrm{s}$. The message payload is equal to 32 bytes. The total transmission time of a broadcast message, including all physical and MAC layer overhead, is set equal to $150 \mu \mathrm{s}$. Considering that each station must wait for a time DIFS $=50 \mu$ s before accessing the channel, the total transfer delay of a message (excluding the time spent in contention) is equal to $T=10$ slots. Notice that broadcast messages are not acknowledged and are never retransmitted, thus they are lost in case of collision.

\subsection{Homogeneous occupation}

We first case consider that all spatial positions along the road are occupied by vehicles, i.e., $\rho=1$, and evaluate the

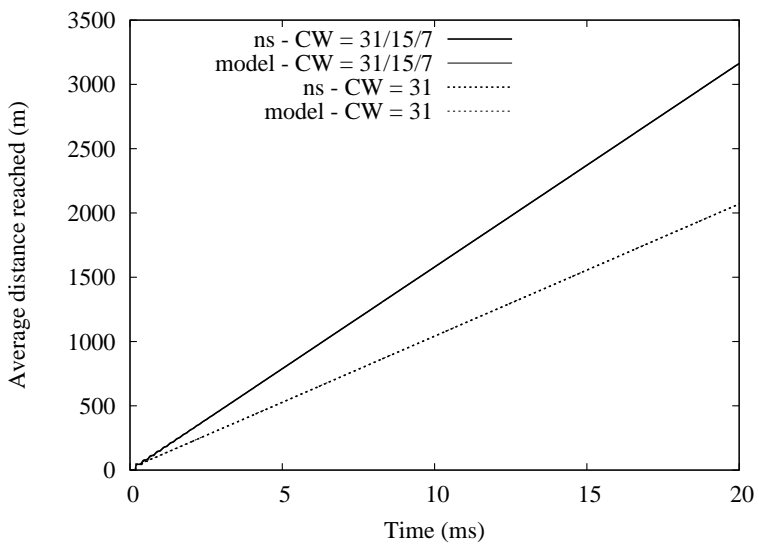

Figure 3. Average value of the maximum distance reached by the message as a function of time when $\rho=1$, for two different contention window schemes.

performance of the proposed broadcast mechanism by using the Gaussian approximation presented in Section 7.1. In this case the message is never blocked because of lack of connectivity $\left(P_{B}[y]=0, \forall y\right)$, and eventually reaches all positions with probability 1 . In this case the Gaussian approximation produces excellent results, almost indistinguishable from simulation results as soon as the message has traveled a few hops.

In Figure 3 we compare the average value of the maximum distance $(E[M(n)]$ in (10)) reached by the message as a function of time in two different cases: (i) fixed contention window $W=31$; (ii) spatial differentiation according to the following rule, hereinafter called " $31 / 15 / 7$ " scheme: $W_{l}=31$ if $1 \geq l \geq 3 ; W_{l}=15$ if $4 \geq l \geq 6$; $W_{l}=7$ if $7 \geq l \geq 9$. In the plot, the curves representing analytical results are overlapped with the ones referring to simulation results, thus the two sets of curves cannot be distinguished. We observe that the spatial differentiation approach outperforms the other scheme, making the propagation of the broadcast message along the road faster.

Figure 4 reports the approximation of the marginal probability $\bar{P}_{R}[y, n]$ sampled every 50 slots (or, equivalently, $1 \mathrm{~ms}$ ). In the plot, there are multiple curves, each of them corresponding to a different sampling time. We observe that the Gaussian approximation is very accurate already after 1 ms (first peak). As expected, the variance of the distribution increases with the passing of time, and the model captures this behavior perfectly.

Next we consider the more complex case of $\rho<1$, under the assumption that the occupation probability is homogeneous along the road. We first study the block probability $P_{B}[y]$, which is a static metric that does not depend on the access scheme employed but only on the occupation proba- 


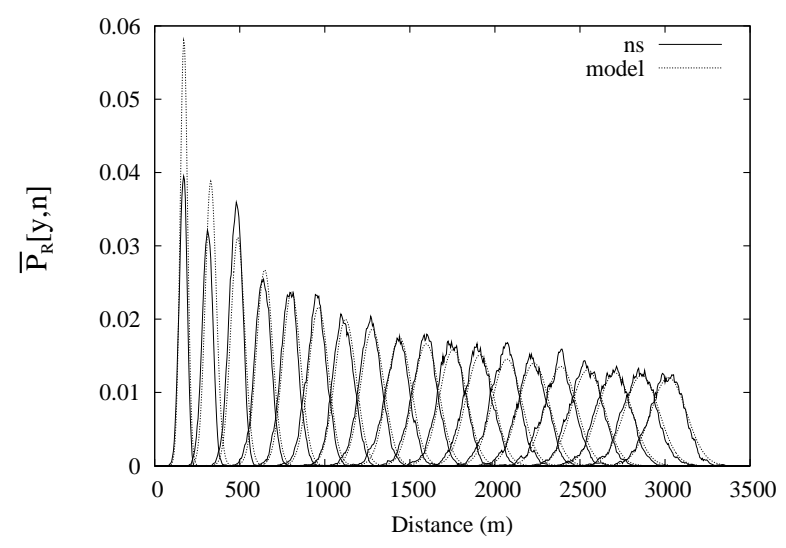

Figure 4. Approximation of the marginal probability of first reception, $\bar{P}_{R}[y, n]$, sampled every 50 slots $=1 \mathrm{~ms}$.

bility $\rho$. Figure 5 compares the block probability predicted by the model (Section 6) and the one measured by simulation, for four different values of $\rho$. The agreement is excellent for all values of $y$, since (3) is exact under the hypothesis that the message stops propagating only because of lack of connectivity, which is indeed the case. Except close to the source, we observe that under homogeneous occupation probability the block probability decays approximately geometrically with the distance.

Next we focus on the case $\rho=0.3$, and compare the delivery delay of the broadcast message under different access schemes. Again, we use the Gaussian approximation as explained in Section 7.1.

Figure 6 reports the average value of the maximum distance $(E[M(n)]$ in (10)) reached by the message as a function of time, for the two access schemes already considered in Figure 3. Since the message soon or later stops propagating because of lack of connectivity, the maximum distance saturates to a maximum value independent of the access scheme, at about $350 \mathrm{~m}$ from the source. We also observe that spatial differentiation still provides a significant advantage with respect to the case of a fixed window $W=31$.

Finally, in Figure 7 we plot the distribution that approximates $\bar{P}_{R}[y, n]$ for the same scenario, sampled every 50 slots. Notice that this probability is not null only if the network is indeed connected up to position $y$, thus a simple Gaussian approximation alone provides a good prediction.

\subsection{Inhomogeneous occupation}

We now consider the more general case in which the occupation probability is non-uniform along the road, but can have an arbitrary distribution. In this case, the Gaussian approximation is no longer applicable and we have to solve

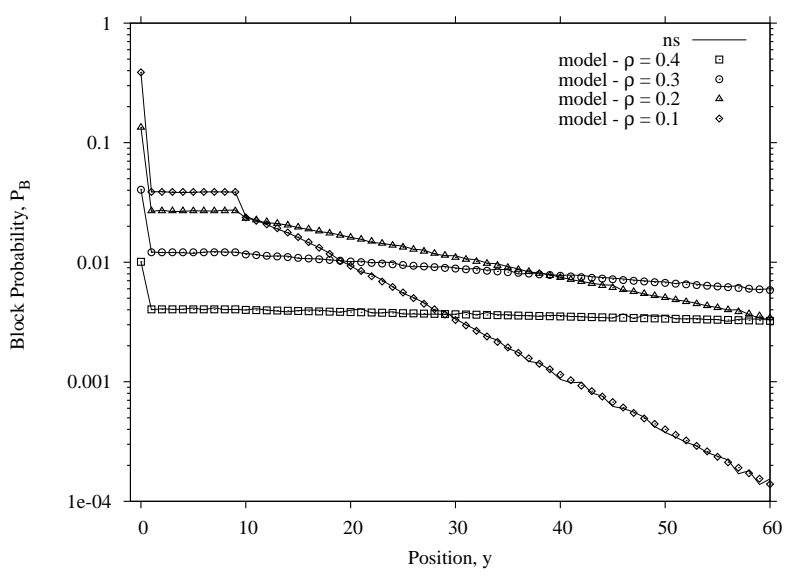

Figure 5. Block probability for four different values of occupation probability $\rho$.



Figure 6. Average value of the maximum distance reached by the message as a function of time when $\rho=0.3$, for two different contention window schemes.

the system using the recursive equations introduced in Section 7 . The block probability $P_{B}[y]$, instead, can be still computed following the simple approach in Section 6.

Consider the following scenario of practical interest: an alarm is generated in a portion of the road having high occupation probability (for instance, this could be due to an accident or traffic congestion), and starts propagating towards regions characterized by a progressively smaller vehicle density. In particular, let's assume that $\rho_{y}=0.8 \cdot 0.99^{y}$, i.e., the occupation probability geometrically decays with the distance from the source of the broadcast message.

Figure 8 compares the analytical prediction with simulation, confirming the accuracy of the analysis. We observe that the block probability reaches a maximum value at about $y \approx 110$ (or, $550 \mathrm{~m}$ from the source). This behavior is due to the fact that close to the source the occupation probabil- 




Figure 7. The approximation of the marginal probability of first reception $\bar{P}_{R}[y, n]$, sampled every $\mathbf{5 0}$ slots in case of homogeneous $\rho=0.3$.

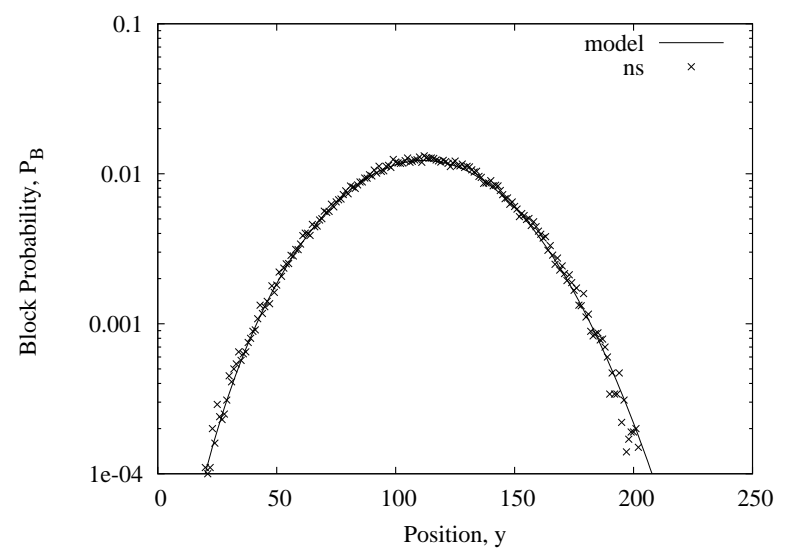

Figure 8. Block probability $P_{B}[y]$ in the case of inhomogeneous occupation probability.

ity is quite high (around 0.8), and the block probability is negligible. The block probability also tends to vanish for $y \rightarrow \infty$, as the probability that the message reaches large distances becomes smaller and smaller.

Finally, Figure 9 presents the marginal probability of first reception regardless of time $\left(\bar{P}_{R}[y, h]\right)$. We show the probability that a vehicle in $y$ receives the message for the first time in $h=5$ and $h=15$ hops. We plot the results obtained when the " $31 / 15 / 7$ " scheme is applied, and compare them with the performance attained with a fixed contention window $W=15$. From Figure 9 we can see that, fixed the number of hops, the spatial differentiation approach allows to reach further distances, i.e., it significantly reduces the number of hops needed to broadcast the message over the road. This implies that, given the vehicle coverage range, less transmissions are needed to disseminate the message and, as a consequence, the broadcast delivery delay decreases.

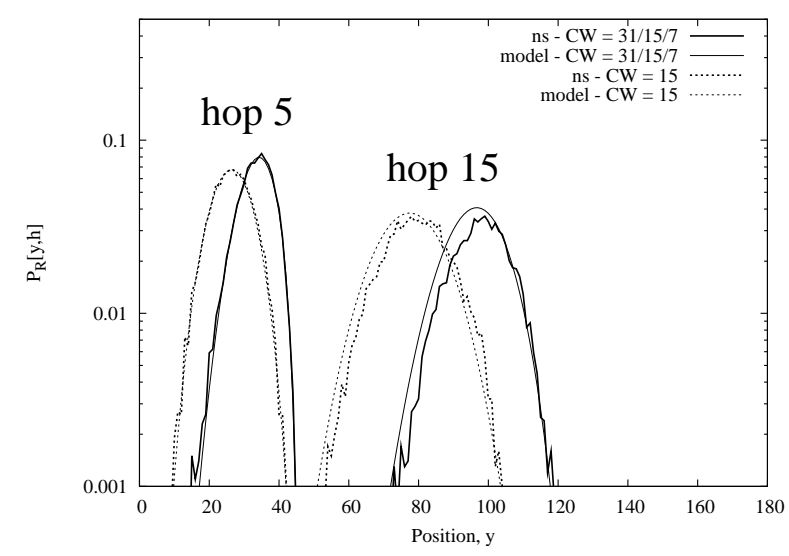

Figure 9. The marginal probability of first reception regardless of time, $\bar{P}_{R}[y, h]$, sampled every 20 slots.

\section{Conclusions}

We developed an application and a channel access mechanisms to provide efficient broadcasting of safety messages in multihop vehicular networks. We presented an analytical framework that allows to derive several metrics of interest such as the message block probability and the message delivery delay. The analytical models were validated against detailed simulation experiments with $n s$.

\section{References}

[1] G. Korkmaz, E. Ekici, F. Ozguner, and U. Ozguner, "Urban Multi-Hop Broadcast Protocol for Inter-Vehicle Communication Systems," First ACM VANET, Oct. 2004.

[2] M. Torrent-Moreno, D. Jiang, and H. Hartenstein, "Broadcast Reception Rates and Effects of Priority Access in 802.11-Based Vehicular Ad-Hoc Networks," First ACM VANET, Philadelphia, PA, Oct. 2004.

[3] A. Zanella, G. Pierobon, and S. Merlin, "On the Limiting Performance of Broadcast Algorithms over Unidimensional Ad-hoc Radio Networks," WPMC' 04, Abano Terme (Padova), Italy, Sep. 2004. 